\title{
Vigilancia tecnológica en el núcleo costa oriental del lago de la Universidad del Zulia
}

\author{
Technological surveillance in the core east coast of the lake of \\ The Universidad of Zulia
}

Daniela Matos

danielamatos86@gmail.com

Código ORCID: 0000-0003-3607-4397

Universidad del Zulia. Maracaibo, Venezuela
4 Articulo recibido en julio 2019

< Arbitrado en agosto 2019

< Publicado en enero 2020

Resumen

En el artículo se describen los objetivos de la implementación de un sistema de vigilancia tecnológica en el Núcleo Costa Oriental del Lago de la Universidad del Zulia. La investigación se catalogó como descriptiva, con un diseño no experimental, transaccional y de campo. Como unidades de análisis fueron tomadas los profesores que dictan clase en el programa de estudios universitarios supervisados (EUS). Se diseñó un cuestionario con 15 ítems. La validez del instrumento se realizó a través del juicio de expertos. La confiabilidad fue determinada mediante la aplicación de la fórmula de Alfa de Crombach obteniendo un valor de 0,90. Los resultados fueron interpretados a través de la media aritmética. Se concluye alta aplicación de cada uno de las objetivos del sistema de vigilancia tecnológica, donde se toman en consideración anticiparse, reducir riesgos, progresar, innovar y cooperar, controlando de esta manera lo que ocurre en su entorno.

Palabras clave: Anticipar, cooperar, innovar, progresar, reducir riesgos, vigilancia tecnológica

\begin{abstract}
In the article, the objectives of the implementation of a technological surveillance system in the Eastern Coast Core of the Lake of the University of Zulia are described. The research was classified as descriptive, with a non-experimental, transactional and field design. As units of analysis, the teachers who taught in the supervised university study program (EUS) were taken. A questionnaire with 15 items was designed. The validity of the instrument was carried out through expert judgment. Reliability was determined by applying the Crombach's Alpha formula, obtaining a value of 0.90 . The results were interpreted through the arithmetic mean. High application of each of the objectives of the technological surveillance system is concluded, where anticipation, risk reduction, progress, innovation and cooperation are taken into consideration, thus controlling what happens in their environment.
\end{abstract}

Keywords: Anticipate, cooperate, innovate, progress, reduce risks, technological vigilance 


\section{INTRODUCCIÓN}

El mundo organizacional de hoy se caracteriza por una marcada velocidad para la toma de decisiones, una fuerte competencia y una innovación permanente de procesos, productos y servicios, con una premisa de máxima eficiencia y mínimo costo, dentro de parámetros de estándares de calidad y clase mundial. Este escenario implica que las empresas y organizaciones estén buscando permanentemente nuevas técnicas, herramientas, métodos, esquemas, metodologías y desarrollos que permitan mantener su mercado $y$, en lo posible, ampliarlo para su crecimiento.

En este contexto, la vigilancia tecnológica juega un papel importante, al analizar el comportamiento innovador de los competidores directos e indirectos, estudiando todas las fuentes de información y examinando productos existentes en el mercado para observar las competencias tecnológicas predominantes. Tal afirmación toma relevancia al considerar Martínez (2007) que la vigilancia tecnológica se puede entender como un sistema organizado de observación y análisis del entorno, tratamiento y circulación interna de los hechos observados y posterior utilización en la empresa y permite detectar tendencias y tecnologías claves, captar y analizar acciones de los competidores y analizar los últimos desarrollos de los proveedores.

A nivel mundial, los países en desarrollo utilizan la vigilancia como una práctica común para mantenerse actualizado en el aspecto tecnológico, debido a una dependencia existente en el mundo hacia los líderes de la tecnología, Por otro lado, en el resto de los países, como los latinoamericanos, es considerada una práctica incipiente lo que hace necesario crear un estado de conciencia acerca de los cambios del entorno, que permita anticiparse a los mismos. Ante esta realidad, están inmersas las universidades, las cuales -por su propia naturaleza de investigación y enseñanza, juegan un papel fundamental para definir, a través de la propia investigación, los nuevos medios y modos para comunicar el saber, utilizando tecnologías de punta para así desarrollar nuevos procesos de enseñanzas y aprendizajes flexibles.

En consecuencia surgen los estudios universitarios supervisados, las cuales ofrecen la posibilidad de cursar unidades curriculares (asignaturas) del área de formación general, bajo la modalidad de Sistema de Educación a Distancia. Este programa forma parte de uno de los núcleos de la Universidad del Zulia, ubicado en el municipio Cabimas, y que a efectos de esta investigación constituye la unidad de información.

Cabe destacar, que la investigadora a través de entrevistas de carácter informal a los profesores adscritos a esta coordinación de estudios a distancia, evidencia que la principal causa que origina la falta de vigilancia tecnológica dentro de la universidad en estudio se debe a la inexistencia de un departamento de vigilancia como tal, que se rija bajo unos parámetros y directrices establecidas, que cuente con una estructura organizacional, además de contar con un recurso humano especialista en el área. Bajo esta óptica, se presentan los resultados obtenidos al considerar la investigadora la importancia de describir los objetivos de la implementación de un sistema de vigilancia tecnológica en el Núcleo Costa Oriental del Lago de la Universidad del Zulia. 


\section{Sistema de vigilancia tecnológica}

Escorsa y Valls (2005) afirman que vigilancia tecnológica "consiste en realizar de manera sistemática, la captura, el análisis, la difusión y la explotación de las transformaciones técnicas útiles para la supenvivencia y el crecimiento de la empresa. Es decir debe alertar sobre toda innovación científica o técnica susceptible que permita crear oportunidades o amenazas latentes". Por otro lado, Delgado y Arrebato (2011) establecen que la vigilancia tecnológica es un conjunto de acciones coordinadas de búsqueda, tratamiento (filtrado, clasificación, análisis) y distribución de la información obtenida de modo legal, útil para distintas personas de una organización en un proceso de toma de decisiones y para la reflexión estratégica. Asimismo, AENOR (2006) define la vigilancia tecnológica según la norma UNE 16.6006 (2018) como un "proceso organizado, selectivo y sistemático, para captar información del exterior y de la propia organización sobre ciencia y tecnología, seleccionarla, analizarla, difundirla y comunicarla, para convertirla en conocimiento con el fin de tomar decisiones con menor riesgo y poder anticiparse a los cambios".

A efectos de esta investigación, se considera la vigilancia tecnológica como el esfuerzo sistemático y organizado por la empresa de observación, captación, análisis, difusión precisa y recuperación de información sobre los hechos del entorno económico, tecnológico, social o comercial, relevantes para la misma por poder implicar una oportunidad o amenaza para ésta, con objeto de poder tomar decisiones con menor riesgo y poder anticiparse a los cambios.

Hidalgo y otros (2002) identifican algunas de las razones para realizar la vigilancia tecnológica, el detectar oportunidades antes que los competidores, conocer el estado del arte de su dominio empresarial, orientar el desarrollo de nuevos productos, así como también encontrar socios financieros, comerciales y tecnológicos. Estos agrupan las actividades de la siguiente manera:

a) Anticipar: Detectar los cambios. Alertar sobre los cambios o amenazas procedentes del mismo sector de actividades o sectores diferentes a la de la empresa.

b) Reducir Riesgos: Detectar Amenazas. Evitar barreras no arancelarias en mercado exteriores. Comprobar si los demás nos están copiando.

c) Progresar: Detectar los desfases. Detectar oportunidades de oportunidades de inversión y comercialización

d) Innovar: Detectar ideas y nuevas soluciones. Ayudar a decidir el programa de nuevos productos y su estrategia. Contribuir a abandonar a tiempo un determinado proyecto.

e) Cooperar: Conocer nuevos socios. Identificar socios adecuados para desarrollar proyectos conjuntos, ahorrando inversiones. Facilitar la incorporación de nuevos avances tecnológicos a los propios productos y servicios.

Para COTEC (2001) los objetivos de la vigilancia tecnológica se pueden enumerar en cinco (5) categorías que le permitan detectar las oportunidades antes que la competencia, conocer el estado del arte en su dominio empresarial, tomar posición en su sector, orientar la I+D, encontrar socios tecnológicos $y$ financieros. 
a) Anticipar: Detectar los cambios: Alerta sobre cambios o amenazas provenientes de sectores distintos al de la empresa. Los esfuerzos de la vigilancia deben adelantarse en el tiempo con respecto a la competencia.

b) Reducir riesgos: Detectar amenazas. Permite evitar barreras no arancelarias en mercados exteriores. La vigilancia también puede extender sus resultados a aspectos como las barreras técnicas a la distribución de productos. Además permite detectar si las demás empresas se están copiando de los productos de la organización.

c) Progresar: Detectar los desfases. Detecta oportunidades de inversión y comercialización. Su interrupción puede originar pérdida de mercados.

d) Innovar: Detectar ideas y nuevas soluciones. Ayuda a decidir el programa de I+D y su estrategia. Los resultados de la vigilancia pueden ayudar a la dirección a decidir la orientación de sus proyectos de I+D y el enfoque técnico de los mismos. Contribuye a abandonar a tiempo un determinado proyecto de I+D. En ocasiones, la vigilancia puede proporcionar como resultado el abandono de un proyecto de innovación y la liberación de sus recursos hacia otras inversiones más productivas. Estas decisiones requieren por sus costes e insatisfacción a corto plazo de una información sólida.

e) Cooperar: conocer nuevos socios. Identifica socios adecuados en proyectos conjuntos de I+D ahorrando inversiones. La idoneidad de un socio en un proyecto conjunto no solo reduce el esfuerzo económico, sino que también evita en ocasiones la realización de desarrollos paralelos. Facilita la incorporación de nuevos avances tecnológicos a los propios productos y procesos. Es esta una de las funciones más importantes de la vigilancia tecnológica.

Antes de iniciar cualquiera de las anteriores acciones, según COTEC (2001), es conveniente plantearse, a modo de reflexión, las siguientes preguntas:

a) ¿Cuál es el objeto de la vigilancia?

b) ¿Qué debemos vigilar?, ¿QQué informaciones buscar?

c) ¿¿Dónde localizarlas?

d) ¿Cómo tratar y organizar la información?

e) ¿A quién comunicar la información en la empresa?

f) ¿Cómo promover la implicación de todo el personal?

g) ¿QQué recursos vamos a destinar?

A continuación se describen cada una de las interrogantes antes mencionadas.

\section{a) ¿Cuál es el objeto de la vigilancia?}

El objeto principal de la vigilancia es el de balizar el camino que la empresa debe seguir para alcanzar los objetivos trazados por su estrategia. Ahora bien, tales objetivos suelen concretarse en distintos aspectos a los que la vigilancia se puede circunscribir.

\section{b) ¿Qué debemos vigilar?, ¿Qué} informaciones buscar?

La empresa tiene recursos limitados y por ello no puede vigilar todos los aspectos. Además de esto la vigilancia debe ser focalizada, esto es centrada sobre las prioridades que marque la estrategia a medio plazo y las necesidades del día a día, optimizada por razones de coste y tiempo de dedicación. 
Vigilar las tecnologías que pueden incidir en nuestra empresa: por un lado, hay que evitar reinventar la rueda; por otro, hay que tratar de mejorar lo que actualmente se hace, con nuevas aportaciones. En cualquier caso se debe ser consciente que muchas de las amenazas y oportunidades están fuera de nuestro sector Es un hecho que las tecnologías extienden su campo de forma horizontal provocando el solapamiento de muchos sectores industriales y el surgimiento de nuevos campos. Esto, junto a la globalización de la economía, hace que muchas de las amenazas provengan de mercados y sectores ajenos al propio.

\section{c) ¿Dónde localizarlas?}

Los cambios y sucesos que hay que vigilar van emitiendo señales en el tiempo, que un buen sistema de vigilancia debe detectar cuanto antes. Tales señales se difunden a través de distintas fuentes de información o aparecen recogidas en ellas. Para apreciar de forma orientativa cómo un mismo hecho va produciendo señales a lo largo del tiempo, e igualmente cómo las posibilidades de que sean detectadas están ligada a la naturaleza de la fuente de información, se muestra la figura a continuación.

\section{d) ¿Cómo tratar y organizar la información?}

Dado que la vigilancia debe dar como resultado un suministro de información oportuna para la toma de decisiones, es esencial que la cantidad, heterogeneidad y complejidad de las señales y los datos que se recogen, sea filtrada y homogeneizada. En pocas palabras, un sistema de vigilancia debe ser capaz de transformar la abundancia de información en propuestas de acciones y medidas que se han de tomar, con recomendaciones. Sin embargo, dicha actividad sirve de poco, si no se ajusta al patrón de toma de decisiones de los responsables. Igualmente, la información difícilmente recuperable aporta poco valor cuando más se necesita. Además, las decisiones pueden tomarse tiempo después de haberse recogido determinadas señales en el mercado, por lo que será necesario poder recuperar en el momento justo dicha información.

\section{e) ¿A quién comunicar la información en la empresa?}

En los anteriores apartados de este documento hemos visto que la información pasa de ser captada a ser analizada, para posteriormente ser utilizada en la toma de decisiones. Ello nos muestra que obviamente los destinatarios por excelencia de la actividad de vigilancia son aquellos cuya actividad está o puede estar condicionada por el exterior. Dirección, compras, marketing, ventas, $I+D$, etc. son actividades típicas que requieren de información con significado, para su actividad y toma de decisiones. Es, pues, esencial que la información fluya selectivamente por toda la empresa para que llegue a quien la necesita, evitando la saturación.

Uno de los obstáculos para la circulación de la información es la visión parcelada de la empresa que tienen sus distintos efectivos. La manera de mejorar este aspecto pasa, por una parte, por hacer descender $y$ difundir a la plantilla directrices conformes a la estrategia de la empresa para que exista una visión compartida global, para que hasta el último empleado tenga una visión más estratégica de la empresa. Por otra 
parte, pasa por hacer participar en reuniones interdepartamentales así como organizar el plan de carrera de los nuevos empleados en distintos departamentos. Otra medida práctica, de uso en sectores cercanos a la ciencia, para la circulación de información, es la de establecer perfiles de investigación, que con carácter periódico se difunden, sobre temas concretos.

\section{f) ¿̇Cómo promover la implicación de todo el personal?}

Para la Agence Nationale de Valorisation de la Recherche (ANVAR, por sus siglas en francés), una conclusión de su análisis de la innovación y la vigilancia en la empresa está en que, si bien es bueno que algún responsable se encargue de la síntesis de las informaciones recogidas, de la animación y de la continuidad de la práctica de la vigilancia, esta debe estar participada por todos en la empresa. Cada uno, desde su dominio, desde su entorno, es un receptor potencial; es decir es responsable de lo suyo.

A ello se suma que la convergencia de diversas informaciones desde diversos puntos de vista, es lo que aporta valor añadido y enriquece la síntesis y el análisis. La vigilancia muchas veces no exige una estructura suplementaria, sino una mayor coherencia de lo que actualmente existe, a través de una buena comunicación interpersonal. Ahora bien, una labor sistemática requiere dedicación y, por tanto, un esfuerzo por parte de la plantilla. Una primera medida en este sentido es el reconocimiento por la dirección del tiempo invertido en tareas de vigilancia.

Para asegurar la participación, la dirección debe prever un sistema de incentivos que en la mayoría de ocasiones no tiene por qué ser de tipo económico. La idoneidad de uno $u$ otro sistema de incentivos depende de la cultura de cada organización. A partir de la experiencia en diversas empresas, se puede extraer unas características comunes para aquellos sistemas de vigilancia tecnológica que funcionan, y que, aunque de sentido común, conviene recordar:

- Definir las responsabilidades de cada miembro de la organización y lo que se espera de él en la vigilancia tecnológica.

- Son posibles los aumentos selectivos de salario, sin implicar cambios en la jerarquía, en función del rendimiento, etcétera.

- Las organizaciones cada vez más planas ofrecen cada vez menos oportunidades de promoción.

- Las alternativas a la retribución deben ser fácilmente comprensibles y cuantificables por todos.

- Las recompensas o agradecimientos del hacho merecedor no deben dilatarse en el tiempo.

- Los incentivos son temporales y reversibles, no una regla establecida. Las recompensas pueden ser variables en función de lo que se dispone.

En una nueva economía basada en el conocimiento, la práctica de la vigilancia contribuye enriqueciendo a la organización con los conocimientos pertinentes a partir de las informaciones filtradas y valorizadas, del exterior. El cambio hacia una cultura «informacional», de manejo e intercambio de información, no es inmediato. Sea cual sea el sistema de incentivos de la empresa, éste debe favorecer cualquier actividad en tal dirección. 


\section{g) ¿Qué recursos vamos a destinar?}

Según los aspectos que hay que vigilar, las fuentes que mejor informan de dichos aspectos, la forma en que se va a filtrar y analizar tales informaciones, las vías de archivo y/o difusión y el personal directamente implicado, se podrá identificar una cantidad de recursos necesarios para cubrirlos.

Por lo antes mencionado es por ello que Escorsa y Maspons (2001) mencionan en primer lugar, que las empresas en general quieren saber en qué tecnologías y en qué productos se está trabajando. Es decir, en el área de su empresa, cuáles son las líneas de investigación, con qué se trabaja, con qué se investiga $y$, por lo tanto, qué se publica y qué se patenta.

Interesa tener bastante claro qué tecnologías emergen, van a más, y qué tecnologías quedan obsoletas y superadas por otras que surgen. Saber qué es lo nuevo que emerge en el área es un tema prioritario, ya que probablemente interesará captarlo cuanto antes mejor. En segundo lugar, interesa conocer qué hacen los competidores, en qué investigan, dónde patentan, por dónde se mueven, para intentar seguirles. $Y$, finalmente, las empresas de una tecnología determinada quieren saber quién es el líder, y en qué instituto, en qué universidad, en qué empresa está, para intentar ponerse en contacto con él para conseguir ventaja.

Los señalamientos antes mencionados de los distintos autores coinciden en líneas generales en establecer cinco razones para la práctica de la vigilancia tecnológica, es decir, categorizan las razones de la misma manera. Lo que para efectos de esta investigación serían las descripciones de los objetivos que persigue la implementación de un sistema de vigilancia tecnológica para las Universidades del municipio Maracaibo que aplican los estudios a distancia. Fijándose posición de esta manera con las razones descritas por la fundación COTEC (2001).

En líneas generales las razones de un sistema de vigilancia tecnológica es el adoptar todos los cambios que se avecinan, que posiblemente resulten en amenazas en oportunidades de crecimiento organizacional, financiero y tecnológico.

\section{MÉTODO}

La investigación se catalogó como descriptiva, con un diseño no experimental, transaccional y de campo. Se determinó una población constituida por 14 profesores correspondientes al Núcleo Costa Oriental del Lago de la Universidad del Zulia específicamente los que dictan clase en el programa de estudios universitarios supervisados (EUS).

La técnica de recolección de datos fue un cuestionario constituido por 15 ítems con posibilidades de respuestas tipo frecuencial, en mismo fue validado a través del juicio de expertos y la confiabilidad determinada mediante la aplicación de la fórmula de Alfa de Crombach, obteniendo un valor de 0,90.

Para el procesamiento de los datos, se utilizó el método de estadística descriptiva, a través de la media aritmética, para su interpretación fue diseñado un baremo a través de una tabla de rango, intervalo y categoría, la cual se muestra en la Tabla 1. 
Tabla 1. Baremo para la interpretación de la media aritmética

\begin{tabular}{|c|c|c|}
\hline INTERVALO & CATEGORÍA & DESCRIPCIÓN \\
\hline $4,21-5,00$ & Muy alta aplicación & \multirow{2}{*}{$\begin{array}{c}\text { Si la aplicación es muy alta o alta se considera una fortaleza } \\
\text { para el sistema de vigilancia tecnológica }\end{array}$} \\
\hline $3,41-4,20$ & Alta aplicación & \\
\hline $2,61-3,40$ & Moderada aplicación & \multirow{3}{*}{$\begin{array}{c}\text { Si la aplicación es moderada, baja o muy baja se considera } \\
\text { una debilidad para el sistema de vigilancia tecnológica, por } \\
\text { tanto requiere mejoras. }\end{array}$} \\
\hline $1,81-2,60$ & Baja aplicación & \\
\hline $1,00-1,80$ & Muy baja aplicación & \\
\hline
\end{tabular}

Fuente: Elaboración propia

\section{RESULTADOS}

En la Tabla 2 se presenta el promedio reportado para el primer indicador a estudiar denominado anticiparse, obteniendo un resultado de 3,92 indicando alta aplicación, que según el baremo de interpretación, es considerada como una fortaleza para el sistema de vigilancia tecnológica. Los ítem 1, 2 y 3 obtuvieron promedios de 4,$00 ; 3,60$ y 4,15, respectivamente, indicando alta aplicación en cuanto a si: existen mecanismos que permitan mantenerse alerta a los cambios, muestra de actitudes proactivas que permiten convertir amenazas en oportunidades y alerta a nuevos procesos que permitan crear ventajas con respecto a los competidores.

Tabla 2. Indicador: Anticiparse

\begin{tabular}{|c|c|c|c|c|}
\hline & ÍTEM & $\mathrm{X}$ & CATEGORÍA & INTERPRETACIÓN \\
\hline \multicolumn{5}{|c|}{ Considera usted que en la coordinación de estudios universitarios supervisados } \\
\hline 1 & $\begin{array}{l}\text { Existen mecanismos que permitan } \\
\text { mantenerse alerta a los cambios }\end{array}$ & 4,00 & Alta aplicación & $\begin{array}{l}\text { Fortaleza para el sistema de } \\
\text { vigilancia tecnológica }\end{array}$ \\
\hline 2 & $\begin{array}{l}\text { Hay muestra de actitudes proactivas } \\
\text { que permiten convertir amenazas } \\
\text { en oportunidades }\end{array}$ & 3,60 & Alta aplicación & $\begin{array}{l}\text { Fortaleza para el sistema de } \\
\text { vigilancia tecnológica }\end{array}$ \\
\hline \multirow[t]{2}{*}{3} & $\begin{array}{l}\text { Mantiene alerta a nuevos procesos } \\
\text { que permitan crear ventajas } \\
\text { con respecto a los competidores. }\end{array}$ & 4,15 & Alta aplicación & $\begin{array}{l}\text { Fortaleza para el sistema de } \\
\text { vigilancia tecnológica }\end{array}$ \\
\hline & TOTAL INDICADOR: & 3,92 & $\begin{array}{c}\text { Alta } \\
\text { aplicación }\end{array}$ & $\begin{array}{l}\text { Fortaleza para el sistema de } \\
\text { vigilancia tecnológica }\end{array}$ \\
\hline
\end{tabular}

Fuente: Elaboración propia

Los resultados mostrados, corresponden con los aportes de Hidalgo y otros (2002) para quienes anticipar permite alertar sobre los cambios o amenazas procedentes del mismo sector de actividades o sectores diferentes a la de la empresa. En este sentido, para la investigadora, en los estudios universitarios supervisados del núcleo bajo estudio, adoptar una actitud proactiva permite que todos los cambios y amenazas a priori puedan convertirse en oportunidades. El detectar y disponer de 
manera anticipada otorga una ventaja que si se sabe aprovechar colocara a la organización en primera línea de cara a la satisfacción del cliente.

Según estos hallazgos, el reto de las nuevas aplicaciones es anticiparse a lo que se pueda llegar a necesitar. De manera que, estos sistemas de educación universitaria deben estar alerta a cambios o amenazas provenientes de sectores iguales o distintos. Los esfuerzos de la vigilancia deben adelantarse en el tiempo con respecto a la competencia. Anticiparse a los cambios puede hacer la diferencia en términos competitivos al favorecer a una universidad para adelantarse a la competencia.
En cuanto al análisis del indicador reducir riesgos, en la Tabla 3, se evidencia que existe alta aplicación del mismo con una media de 3,58, indicando una fortaleza para para el sistema de vigilancia tecnológica. Al detalle se observa, alta aplicación para todos los ítems relacionados, por tanto: se analizan si los competidores están copiando sus productos $(3,43)$, analizan si los competidores están copiando sus procesos $(3,50) y$, finalmente, se determina a priori los inconvenientes que pueda presentarse con relación al uso de la tecnología $(3,82)$.

Tabla 3. Indicador: Reducir riesgos

\begin{tabular}{|c|c|c|c|c|}
\hline & ÍTEM & $x$ & CATEGORÍA & INTERPRETACIÓN \\
\hline \multicolumn{5}{|c|}{ Considera usted que en la coordinación de estudios universitarios supervisados } \\
\hline 4 & $\begin{array}{l}\text { Se analizan si los competidores } \\
\text { están copiando sus productos }\end{array}$ & 3,43 & Alta aplicación & $\begin{array}{l}\text { Fortaleza para el sistema de } \\
\text { vigilancia tecnológica }\end{array}$ \\
\hline 5 & $\begin{array}{l}\text { Se analizan si los competidores } \\
\text { están copiando sus procesos }\end{array}$ & 3,50 & Alta aplicación & $\begin{array}{l}\text { Fortaleza para el sistema de } \\
\text { vigilancia tecnológica }\end{array}$ \\
\hline \multirow[t]{2}{*}{6} & $\begin{array}{l}\text { Se determina a priori los } \\
\text { inconvenientes que pueda presentarse } \\
\text { con relación al uso de la tecnología }\end{array}$ & 3,82 & Alta aplicación & $\begin{array}{l}\text { Fortaleza para el sistema de } \\
\text { vigilancia tecnológica }\end{array}$ \\
\hline & TOTAL INDICADOR: & 3,58 & $\begin{array}{l}\text { Alta } \\
\text { aplicación }\end{array}$ & $\begin{array}{l}\text { Fortaleza para el sistema de } \\
\text { vigilancia tecnológica }\end{array}$ \\
\hline
\end{tabular}

Fuente: Elaboración propia

En este sentido, los resultados se encuentran alineados con lo expuesto por Hidalgo y otros (2002) quienes consideran que detectar amenazas permite evitar barreras no arancelarias en mercados exteriores. A este respecto, la investigadora considera, que la vigilancia tiene dos facetas una que supone el hacer frente a amenazas y oportunidades $y$, otra, el seguimiento del entorno. El riesgo constituye una falta de conocimiento sobre futuros acontecimientos, se puede definir como el efecto acumulativo que estos acontecimientos adversos podrían tener sobres los objetivos de la actividad planeada.

En lo que respecta al indicador progresar, se tiene una media de 4,25, tal como se muestra en la Tabla 4, indicando alta aplicación en el sistema de vigilancia tecnológica, otorgándoles fortalezas. Al 
detalle se observa, alta aplicación de las actividades relacionadas a: se detecta oportunidades de inversión $(4,30)$, se detecta oportunidades comerciales $(4,20) \quad y$, finalmente, se evalúan las tecnologías para adaptarlas a requerimientos particulares $(4,25)$.

Tabla 4. Indicador: Progresar

\begin{tabular}{cccc}
\hline ÍTEM & X & CATEGORÍA & INTERPRETACIÓN \\
\hline Considera usted que en la coordinación de estudios universitarios supervisados \\
7 Se detecta oportunidades de inversión. & 4,30 & Alta aplicación & $\begin{array}{c}\text { Fortaleza para el sistema de } \\
\text { vigilancia tecnológica }\end{array}$ \\
8 Se detecta oportunidades comerciales & 4,20 & Alta aplicación & $\begin{array}{c}\text { Fortaleza para el sistema de } \\
\text { vigilancia tecnológica }\end{array}$ \\
$\begin{array}{c}\text { Se evalúan las tecnologías para } \\
\text { adaptarlas a requerimientos particulares }\end{array}$ & 4,25 & Alta aplicación & $\begin{array}{c}\text { Fortaleza para el sistema de } \\
\text { vigilancia tecnológica }\end{array}$ \\
TOTAL INDICADOR: & 4,25 & $\begin{array}{c}\text { Alta } \\
\text { aplicación }\end{array}$ & $\begin{array}{c}\text { Fortaleza para el sistema de } \\
\text { vigilancia tecnológica }\end{array}$ \\
\hline
\end{tabular}

Fuente: Elaboración propia (2019)

Visto así, se tiene alta congruencia con lo establecido por Hidalgo y otros (2002) al considerar que el objetivo de la vigilancia es detectar oportunidades de oportunidades de inversión y comercialización. Bajo estas consideraciones, para la investigadora el núcleo en sus estudios universitarios supervisados detecta oportunidades de inversión y comercialización ya que su interrupción puede originar pérdida de mercados y futuro declive de la universidad.

En tal sentido, los procesos de vigilancia tecnológica permiten a las universidades descubrir oportunidades de inversión y negocios. Los beneficios que trae consigo la aplicación de dichos procesos dependen en gran medida del compromiso por parte de estos institutos.

En la Tabla 5, mostrada para analizar el comportamiento del indicador innovar, se evidencia una media de 4,08 indicando alta aplicación de este indicador otorgándole fortaleza para el sistema de vigilancia tecnológica. Al detallar por ítem se observa, alta aplicación de que: se generan nuevas ideas a partir de uso de la vigilancia tecnológica $(4,00)$, se promueven proyectos innovadores dirigidos hacia nuevos productos $(4,05)$ y se detecta el momento oportuno para abandonar un proyecto de I+D que no genera valor agregado $(4,20)$. 
Tabla 5. Indicador: Innovar

ITEM

X CATEGORÍA

INTERPRETACIÓN

Considera usted que en la coordinación de estudios universitarios supervisados

10 Se generan nuevas ideas a partir de uso de la vigilancia tecnológica.

11 Se promueven proyectos innovadores dirigidos hacia nuevos productos.

Se detecta el momento oportuno para

12 abandonar un proyecto de I+D que no genera valor agregado.

TOTAL INDICADOR:
4,00 Alta aplicación

4,05 Alta aplicación

4,20 Alta aplicación

4,08
Fortaleza para el sistema de vigilancia tecnológica

Fortaleza para el sistema de vigilancia tecnológica

Fortaleza para el sistema de vigilancia tecnológica

Fortaleza para el sistema de vigilancia tecnológica

Fuente: Elaboración propia

Lo evidenciado alcanza alta congruencia con Hidalgo y otros (2002) cuando afirman que detectar ideas y nuevas soluciones, ayudar a decidir el programa de nuevos productos y su estrategia. Por lo que, para la investigadora, innovar puede ayudar a las autoridades a decidir la orientación de sus proyectos y el enfoque técnico de los mismos. De esta manera, innovar como objetivo de la vigilancia tecnológica, puede traer consigo el abandono de un proyecto de innovación y la liberación de sus recursos hacia otras inversiones más productivas por parte de las universidades.

Seguidamente, se presentan en la Tabla 6 los resultados para el último indicador de la dimensión, denominado cooperar. Se observa una media de 4,11, indicando alta aplicación otorgándole fortaleza para el sistema de vigilancia tecnológica. Los resultados obtenidos muestran alta aplicación de las actividades medidas por los ítems, de tal forma que en estos estudios se realiza con alta frecuencia lo referido a si: se identifican los socios adecuados para desarrollar proyectos en conjunto $(4,10)$, se facilita el ingreso de nuevas tecnologías para mejorar sus procesos $(4,12)$ y, finalmente, se facilita el ingreso de nuevas tecnologías para mejorar sus servicios $(4,12)$.

Tabla 6. Indicador: Cooperar

ÍTEM $\mathrm{X}$ CATEGORÍA INTERPRETACIÓN

Considera usted que en la coordinación de estudios universitarios supervisados

13 Se identifican los socios adecuados para desarrollar proyectos en conjunto.

4,10 Alta aplicación

4,12 Alta aplicación

14 Se facilita el ingreso de nuevas tecnologías para mejorar sus procesos.

15 Se facilita el ingreso de nuevas tecnologías para mejorar sus servicios.
4,12

TOTAL INDICADOR:
4,11
Fortaleza para el sistema de vigilancia tecnológica

Fortaleza para el sistema de vigilancia tecnológica

Fortaleza para el sistema de vigilancia tecnológica

\footnotetext{
Fuente: Elaboración propia
} 
De acuerdo a los resultados expuestos para el indicador cooperar, catalogado de alta aplicación, se muestra alto nivel de congruencia con lo planteado por Hidalgo y otros (2002) al indicar como objetivo de la vigilancia tecnología el identificar socios adecuados para desarrollar proyectos conjuntos, ahorrando inversiones, facilita la incorporación de nuevos avances tecnológicos a los propios productos y servicios.

Para la investigadora, identificar los socios adecuados en proyectos conjuntos, permite ahorrar inversiones ya que la idoneidad de un socio en un proyecto conjunto no solo reduce el esfuerzo económico, sino que también evita en ocasiones la realización de desarrollos paralelos; por otro lado facilita la incorporación de nuevos avances tecnológicos a los propios productos y procesos.

Lo anteriormente citado, es un fiel reflejo de lo que ha representado la cooperación por parte de las universidades, permitiendo de esta manera a las instituciones responder a las demandas del entorno en conjunto con otras organizaciones de la rama, lo que conlleva a cooperaciones desde el punto de vista organizativo, comercial y tecnológico.

La Tabla 7 muestra los resultados correspondientes a la dimensión objetivos de la vigilancia tecnológica, en la cual se ve reflejada una media de 3,99, considerada de alta aplicación, indicando fortalezas para el sistema de vigilancia tecnológica.

Tabla 7. Dimensión: objetivos de la vigilancia tecnológica

\begin{tabular}{|c|c|c|c|}
\hline INDICADOR & $x$ & CATEGORÍA & INTERPRETACIÓN \\
\hline Anticiparse & 3,92 & Alta aplicación & $\begin{array}{c}\text { Fortaleza para el sistema de } \\
\text { vigilancia tecnológica }\end{array}$ \\
\hline Reducir riesgos & 3,58 & Alta aplicación & $\begin{array}{l}\text { Fortaleza para el sistema de } \\
\text { vigilancia tecnológica }\end{array}$ \\
\hline Progresar & 4,25 & Alta aplicación & $\begin{array}{l}\text { Fortaleza para el sistema de } \\
\text { vigilancia tecnológica }\end{array}$ \\
\hline Innovar & 4,08 & Alta aplicación & $\begin{array}{l}\text { Fortaleza para el sistema de } \\
\text { vigilancia tecnológica }\end{array}$ \\
\hline Cooperar & 4,11 & Alta aplicación & $\begin{array}{l}\text { Fortaleza para el sistema de } \\
\text { vigilancia tecnológica }\end{array}$ \\
\hline DIMENSIÓN: & 3,99 & Alta aplicación & $\begin{array}{l}\text { Fortaleza para el sistema de } \\
\text { vigilancia tecnológica }\end{array}$ \\
\hline
\end{tabular}

Fuente: Elaboración propia 
Lo anterior posee alta coincidencia con lo propuesto por Hidalgo y otros (2002) para quienes identificar algunas de las razones para realizar la vigilancia tecnológica, permite detectar oportunidades antes que los competidores, conocer el estado del arte de su dominio empresarial, orientar el desarrollo de nuevos productos, así como también encontrar socios financieros, comerciales y tecnológicos.

De esta manera, a criterio de la investigadora la vigilancia tecnológica representa el esfuerzo sistemático y organizado por el núcleo de observación, captación, análisis, difusión precisa y recuperación de información sobre los hechos del entorno económico, tecnológico, social o comercial, relevantes para la misma por poder implicar una oportunidad $\mathrm{O}$ amenaza para ésta, con objeto de poder tomar decisiones con menor riesgo y poder anticiparse a los cambios.

\section{CONCLUSIONES}

En lo que representa el objetivo orientado a describir los objetivos de la implementación de un sistema de vigilancia tecnológica en el Núcleo Costa Oriental del Lago de la Universidad del Zulia, en sus estudios universitarios supervisados, se concluye: alta aplicación de los objetivos de un sistema de vigilancia tecnológica, donde se toman en consideración anticiparse, reducir riesgos, progresar, innovar y cooperar, controlando de esta manera lo que ocurre en su entorno, lo que otorga mayor capacidad de reacción a los cambios que se están produciendo o se pueden producir.

\section{REFERENCIAS}

AENOR (2006). Norma Española Experimental UNE 166006 Gestión de la I+D+i: Sistema de Vigilancia Tecnológica. Disponible en: https://scielo.conicyt.cl/scielo.php?script $=$ sci_nlinks\&ref $=2929879 \&$ pid $=$ S0718 $-5006201600020000300001 \&$ lng $=e s$

COTEC. (2001). Gestión de la innovación y la tecnología en la empresa. Informes sobre el sistema español de innovación. Fundación Cotec para la Innovación Tecnológica. Disponible en: http://ve.scielo.org/scielo.php?script $=$ sc i nlinks\&ref $=1832771$ \&pid $=$ S1012$1587200700030000300002 \&$ Ing $=$ es

Delgado, M., y Arrebato, L., (2011). Diagnóstico integrado de la vigilancia tecnológica en organizaciones. Ingeniería Industrial, 1-6. Disponible en:

https://scielo.conicyt.cl/scielo.php? script $=$ sci nlinks\&ref $=2929885 \&$ pid $=$ S0718 $-5006201600020000300007 \&$ lng $=e s$

Escorsa, P. y Valls, J (2005). Tecnología e innovación en la empresa. Editorial alfaomega. México D.F

Escorsa, P; y Maspons, R. (2005). De la vigilancia tecnológica a la inteligencia a la inteligencia competitiva. Editorial Prentice Hall. Madrid

Hidalgo, A; Serrano, G; Pabon, J. (2002).La gestión de la innovación y la tecnología en las organizaciones. Editorial Pírame, Madrid

Martínez, L (2007). La información como herramienta de la vigilancia estratégica. Revista Iberoamericana sobre usuarios de información, 1-10. Disponible en: https://scielo.conicyt.cl/scielo.php? script $=$ sci_nlinks\&ref $=2929895 \&$ pid $=$ S0718 $-5006201600020000300016 \&$ lng $=e s$ 